\title{
Genus Cosmarium Corda from Thrissur Kole Lands, Kerala
}

\author{
P. Tessy Paul1*, R. Sreekumar ${ }^{2}$ \\ 'Department of Botany, Christ College, Irinjalakuda, Thrissur, Kerala, India, 'Department of Botany, Maharajas College, Ernakulam, \\ Kerala, India
}

Received: 20.10 .2015

Accepted: 12.11 .2015

Published: 13.11 .2015

*Address for correspondence:

P. Tessy Paul,

Department of Botany,

Christ College, Irinjalakuda,

Thrissur - 680 125,

Kerala, India.

Phone: +91-9446233104

E-mail: tessyjohnt@gmail.

com

\begin{abstract}
The present investigation deals with 68 taxa of Cosmarium Corda collected from the Kole lands of Thrissur, Kerala. The Kole lands constitute part of Vembanad-Kol, which is declared as one of the Ramsar sites of Kerala. All the taxa are systematically described with illustrations and their distribution in India. Of these, 6 taxa are the $1^{\text {st }}$ time reported from India and 29 taxa are new additions to the algal flora of Kerala. Cosmarium geminatum Lund. var. ornatum Behre, Cosmarium indentatum Gronbl. var. ellipticum Scott and Gronbl., Cosmarium pseudoconnatum Nordst. var. constrictum West, Cosmarium quadrifarium Lund., Cosmarium quadriverrucosum West and West var. undulatum Scott and Prescott and Cosmarium subturgidum (Turn.) Schmidle are the new additions to the desmid flora of India.
\end{abstract}

KEY WORDS: Cosmarium, desmid, Kerala, Thrissur Kole lands, Vembanad-Kol

\section{INTRODUCTION}

The Kole lands form a unique aquatic ecosystem and are the rice granary of Thrissur and Malappuram districts, Kerala (Johnkutty and Venugopal, 1993). The Kole lands of Kerala constitute part of Vembanad-Kol, which is declared as one of the Ramsar sites of India in 2002 and got international importance (http: / / www.ramsar.org). The Kole lands geographically distributed in Mukundapuram, Chavakkad and Thrissur taluks of Thrissur district, Kerala are designated as “Thrissur Kole." The present manuscript deals with the systematic account, seasonal and spatial variation of Cosmarium Corda from the Kole lands of Thrissur, Kerala.

Cosmarium Corda comes under the family Desmidiaceae of the order Zygnematales and the class Chlorophyceae. The Cosmarium was reported from different parts of India and are distributed in Kerala (Jose and Francis, 2007; Maya et al., 2000; Shaji et al., 1988; Shaji et al., 1989; Shaji and Patel, 1990; Shaji and Patel, 1991; Sheeba and Ramanujan, 2005; Sindhu and Panikkar, 1994; Sindhu and Panikkar, 1995; Suxena et al., 1973), Andhra Pradesh (Suxena and Venkareswarlu, 1966), Bengal (Turner, 1892), Bihar (Das et al., 1990; Saha and Choudhary, 1984), Chhattisgarh (Toppo and Suseela, 2009), Gujarat (Asokakumar and
Patel, 1988; Asokakumar and Patel, 1990), Karnataka (Gurudeva et al., 1983; Hegde, 1986; Hegde and Isaacs, 1988), Maharashtra (Dhande and Jawale, 2009; Freitas and Kamat, 1979; Kamat, 1975a; Kamat, 1975b), Madhya Pradesh (Agarkar et al., 1983; Agarkar et al., 1979; Agarkar and Agarkar, 1977), Tamil Nadu (Perumal and Anand, 2008), and Uttar Pradesh (Chaturvedi et al., 1987; Tiwari and Chauhan, 2007).

\section{MATERIALS AND METHODS}

The present paper is based on the collections of water samples from the Kole lands of Thrissur district, Kerala, during February 2005-January 2006. The study area lies between $10^{\circ} 20^{\prime} 28^{\prime \prime}-10^{\circ} 30^{\prime} 17^{\prime \prime}$ North latitudes and $76^{\circ} 06^{\prime} 31^{\prime \prime}-76^{\circ} 15^{\prime} 26^{\prime \prime}$ East longitudes. The surface water samples were collected monthly from 10 stations namely Pullur, Muriyad, Nambiankavu, Mapranam, Chemmanda, Cherpu, Palakkal, Chettupuzha, Anthikkad and Enamavu. The samples collected were preserved in $4 \%$ formalin solution. The Cosmarium taxa were identified with the help of monographs and research publications (Turner, 1892; Croasdale and Gronblad, 2007; Nurul and Irfanullah, 2006; Scott and Prescott, 1961; West and West, 1905; West and West, 1908; West and West, 1912). The Camera Lucida drawings of all taxa were prepared. 


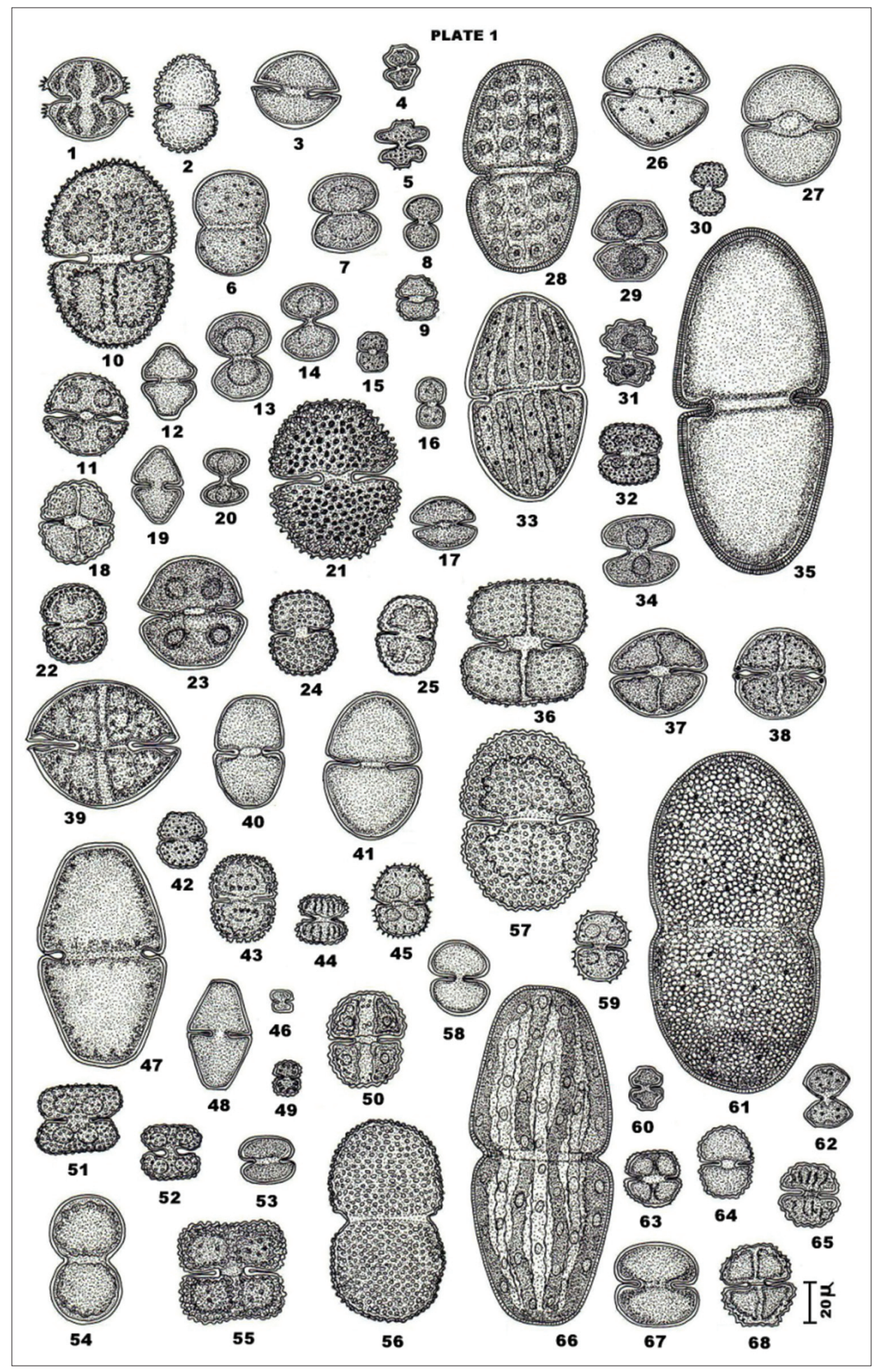

Plate 1 (Figs. 1-68): 1. Cosmarium auriculatum 2. C. binum 3. C. circulare 4. C. clepsydra var. duplex 5. C. commissurale 6. C. connatum 7. C. contractum var. ellipsoideum 8. C. contractum var. minutum 9. C. impressulum 10. C. brebissonii 11. C. cuneatum 12. C. granatum var. nordstedtii 13. C. contractum 14. C. contractum var. incrassatum 15. C. meneghinii 16. C. exiguum 17. C. apertum 18. C. cyclicum 19. C. granatum 20. C. indentatum var. ellipticum 21. C. decoratum var. dentiferum 22. C. arnellii 23. C. lundellii 24. C. margaritatum fa. minor 25. C. manipurence 26. C. Iundellii var. corruptum 27. C. lundellii var. ellipticum 28. C. javanicum 29. C. nitidulum 30. C. portianum 31. C. notabile 32. C. pseudobroomei 33. C. maculatiforme 34. C. pseudotoxicandrum 35. C. maculatum 36. C. margaritatum var. sublatum 37. C. obsoletum 38. C. nudum 39. C. obsoletum var. sitvense 40. C. pseudopyramidatum var. oculatum 41. C. pachydermum 42. C. punctulatum var. subpunctulatum 43 . C. quadrifarium 44. C. geminatum var. ornatum 45. C. quadriverrucosum var. undulatum 46. C. pygmaeum 47. C. pyramidatum 48. C. pyramidatum var. stephanii 49. C. blyttii 50. C. speciosum 51. C. quadrum 52. C. reniforme 53. C. depressum 54. C. pseudoconnatum var. constrictum 55 . C. scabrum 56. C. striolatum 57. C. subspeciosum var. validius 58. C. tumidum 59. C. trachypleurum var. nordstedtii 60. C. retusiforme 61. C. subturgidum 62. C. tjibenongense fa. minus 63. C. spinuliferum 64. C. subcrenatum 65. C. subcostatum 66. C. turgidum 67. C. taxichondrum var. nudum 68. C. vexatu 


\section{RESULTS AND DISCUSSION}

Cosmarium cells are longer than broad, constricted in the middle, semicells very variable in outline, elliptical, subcircular, semicircular, subquadrate, or truncatepyramidate, apex rounded, truncate or subtruncate (West andWest, 1905). During the present investigation, 68 taxa of Cosmarium have been identified from the Kole lands of Thrissur, Kerala and are described systematically with their distribution in India. Of these 6 taxa are new reports to India and 29 taxa are recorded for the $1^{\text {st }}$ time from Kerala. The measurements of length (L) and breadth (B) of the taxa are given in microns $(\mu \mathrm{m})$.

\section{GENUS: COSMARIUM CORDA}

\section{Systematic Account}

1. Cosmarium apertum Turn. (pl. 1, fig. 17)

Turner, 1892. p. 54, pl. 8, fig. 11.

L: 31-34 $\mu \mathrm{m}, \mathrm{B}: 36-39 \mu \mathrm{m}$. Distribution: Gujarat (Asokakumar and Patel, 1988), East India (Turner, 1892); Tamil Nadu (Perumal and Anand, 2008).

2. Cosmarium arnellii Boldt. (pl. 1, fig. 22)

West and West, 1908. vol. 3, p. 205, pl. 84, fig. 11.

L: 39-50.6 $\mu \mathrm{m}, \mathrm{B}: 35-40 \mu \mathrm{m}$. Distribution: East India (Turner, 1892), Tamil Nadu (Perumal and Anand, 2008).

3. Cosmarium auriculatum Reinsch (pl. 1, fig. 1)

Scott and Prescott, 1961. p. 54, pl. 26, fig. 4.

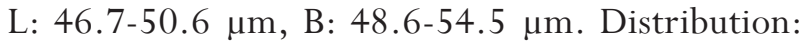
Kerala (Shaji et al., 1988; Sindhu and Panikkar, 1995); Bengal (Turner, 1892); Bihar (Saha and Choudhary, 1984); Gujarat (Asokakumar and Patel, 1990); Karnataka (Hegde and Isaacs, 1988); Maharashtra (Freitas and Kamat, 1979; Kamat, 1975b); Tamil Nadu (Perumal and Anand, 2008).

4. Cosmarium binum Nordst. (pl. 1, fig. 2)

West and West, 1908. vol. 3, p. 246, pl. 88, figs. 10-14. L: 68-85.6 $\mu \mathrm{m}, \mathrm{B}:$ 35-39 $\mu \mathrm{m}$. Distribution: Kerala (Shaji et al., 1988); Bihar (Das et al., 1990); Gujarat (Asokakumar and Patel, 1988); Maharashtra (Freitas and Kamat, 1979; Kamat 1975a); Madhya Pradesh (Agarkar et al., 1983; Agarkar and Agarkar, 1977); Tamil Nadu (Perumal and Anand, 2008).

5. Cosmarium blyttii Wille (pl. 1, fig. 49)

West and West, 1908. vol. 3, p. 225, pl. 86, figs. 1-4. L: 11.7-18.5 $\mu \mathrm{m}, \mathrm{B}: 8.8-15.6 \mu \mathrm{m}$. Distribution: Chhattisgarh (Toppo and Suseela, 2009); Tamil Nadu (Perumal and Anand, 2008).

6. Cosmarium brebissonii Menegh. (pl. 1, fig. 10)

West andWest, 1908. vol. 3, p. 161, pl. 79, figs. 10, 11.
L: 65-97.3 $\mu \mathrm{m}, \mathrm{B}: 49.6-70 \mu \mathrm{m}$. Distribution: Kerala (Sindhu and Panikkar, 1994); East India (Turner, 1892).

7. Cosmarium circulare Reinsch (pl. 1, fig. 3)

West and West, 1905. vol. 2, p. 136, pl. 56, figs. 11, $13,14$.

L: 35-46.7 $\mu \mathrm{m}, \mathrm{B}$ : 39-46.7 $\mu \mathrm{m}$. Distribution: Kerala (Shaji et al., 1989; Sindhu and Panikkar, 1995); Bengal and Central India (Turner 1892); Gujarat (Asokakumar and Patel, 1988); Karnataka (Gurudeva et al., 1983); Uttar Pradesh (Chaturvedi et al., 1987).

8. Cosmarium clepsydra Nordst. var. duplex Prescott (pl. 1, fig. 4)

Shaji and Patel, 1990. pl. 1, fig. 6.

L: 23.3-25.3 $\mu \mathrm{m}, \mathrm{B}: 17.5-18.5 \mu \mathrm{m}$. Distribution: Kerala (Shaji and Patel, 1990); Bihar (Das et al., 1990).

9. Cosmarium commissurale Breb. (pl. 1, fig. 5)

West and West, 1908. vol. 3, p. 154, pl. 78, figs. 11-14. L: 23.3-32 $\mu \mathrm{m}, \mathrm{B}: 35-39 \mu \mathrm{m}$. Distribution: Kerala (Sindhu and Panikkar, 1994).

10. Cosmarium connatum Breb. (pl. 1, fig. 6)

West and West, 1908. vol. 3, p. 25, pl. 67, figs. 15-17. L: 52.5-58.4 $\mu \mathrm{m}, \mathrm{B}: 35-40 \mu \mathrm{m}$. Distribution: Kerala (Sheeba and Ramanujan, 2005; Sindhu and Panikkar, 1995); Gujarat (Asokakumar and Patel, 1988); Maharashtra (Freitas and Kamat, 1979; Kamat, 1975a; Kamat 1975b).

11. Cosmarium contractum Kirchner (pl. 1, fig. 13)

West and West, 1905. vol. 2, p. 170, pl. 61, figs. 23-25. L: 38-42.8 $\mu \mathrm{m}, \mathrm{B}: 25.3-27.2 \mu \mathrm{m}$. Distribution: Kerala (Sheeba and Ramanujan, 2005); Bengal and North India (Turner, 1892); Gujarat (Asokakumar and Patel, 1988); Maharashtra (Kamat, 1975a); Tamil Nadu (Perumal and Anand, 2008).

12. Cosmarium contractum Kirchner var. ellipsoideum (Elfv.) West and West (pl. 1, fig. 7)

West andWest, 1905. vol. 2, p. 172, pl. 61, figs. 28, 35. L: 33-39 $\mu \mathrm{m}, \mathrm{B}: 29-35 \mu \mathrm{m}$. Distribution: Kerala (Suxena et al., 1973); Bihar (Das et al., 1990); Chhattisgarh (Toppo and Suseela, 2009); Gujarat (Asokakumar and Patel, 1988); Maharashtra (Kamat, 1975a).

13. Cosmarium contractum Kirchner var. incrassatum Scott and Prescott (pl. 1, fig. 14)

Scott and Prescott, 1961.p. 56, pl. 27, fig. 5.

L: 33-42.8 $\mu \mathrm{m}, \mathrm{B}: 21.4-27.2 \mu \mathrm{m}$. Distribution: Kerala (Sindhu and Panikkar, 1995); Karnataka (Hegde and Isaacs, 1988).

14. Cosmarium contractum Kirchner var. minutum (Delp.) West and West (pl. 1, fig. 8)

Croasdale and Gronblad, 1964. p. 178, pl. 11, fig. 20. L: 23.3-27.2 $\mu \mathrm{m}, \mathrm{B}: 19.5-21.4 \mu \mathrm{m}$. Distribution: 
Kerala (Shaji et al., 1989); Gujarat (Asokakumar and Patel, 1988); Karnataka (Gurudeva et al., 1983); Maharashtra (Kamat, 1975a; Kamat, 1975b); Madhya Prades (Agarkar et al., 1983).

15. Cosmarium cuneatum Joshua (pl. 1, fig. 11)

Scott and Prescott, 1961. p. 57, pl. 30, fig. 3.

L: 35-41.8 $\mu \mathrm{m}, \mathrm{B}: 38-47.7 \mu \mathrm{m}$. Distribution: Karnataka (Gurudeva et al., 1983); Madhya Pradesh (Agarkar et al., 1983).

16. Cosmarium cyclicum Lund. (pl. 1, fig. 18)

West and West, 1905. vol. 2, p. 145, pl. 58, figs. 8, 9. L: 46.7-50.6 $\mu \mathrm{m}, \mathrm{B}: 42.8-48.6 \mu \mathrm{m}$. Distribution: Bihar (Das et al., 1990).

17. Cosmarium decoratum West et West var. dentiferum West et West (pl. 1, fig. 21)

Shaji et al., 1989. pl. 3, fig. 5.

L: 77.8-85.6 $\mu \mathrm{m}, \mathrm{B}:$ 66-70 $\mu \mathrm{m}$. Distribution: Kerala (Shaji et al., 1989).

18. Cosmarium depressum (Nag.) Lund. (pl. 1, fig. 53)

West and West, 1905. vol. 2, p. 176, pl. 62, figs. 2-5

L: 17-25.2 $\mu \mathrm{m}, \mathrm{B}: 19.5-29.2 \mu \mathrm{m}$. Distribution: East India (Turner, 1892); Tamil Nadu (Perumal and Anand, 2008).

19. Cosmarium exiguum Arch. (pl. 1, fig. 16)

West and West, 1908. vol. 3, p. 63, pl. 70, figs. 17-19. L: 20.4-31 $\mu \mathrm{m}, \mathrm{B}: 12.6-16.5 \mu \mathrm{m}$. Distribution: Kerala (Sindhu and Panikkar, 1994); Andhra Pradesh (Suxena and Venkareswarlu, 1966); East India (Turner, 1892); Maharashtra (Kamat, 1975a); Tamil Nadu (Perumal and Anand, 2008).

20. Cosmarium geminatum Lund. var. ornatum Behre (pl. 1, fig. 44)

Scott and Prescott, 1961. p. 59, pl. 31, fig. 9.

L: 22.4-25.3 $\mu \mathrm{m}, \mathrm{B}: 21.4-24.3 \mu \mathrm{m}$.

21. Cosmarium granatum Breb. (pl. 1, fig. 19)

West and West, 1905. vol. 2, p. 186, pl. 63, figs. 1-3. L: 31-39 $\mu \mathrm{m}, \mathrm{B}: 23.3-27.2 \mu \mathrm{m}$. Distribution: Kerala (Maya et al., 2000); Andhra Pradesh (Suxena and Venkareswarlu, 1966); Bengal (Turner, 1892); Bihar (Das et al., 1990; Saha and Choudhary, 1984); Gujarat (Asokakumar and Patel, 1988); Maharashtra (Dhande and Jawale, 2009; Freitas and Kamat, 1979; Kamat, 1975a); Madhya Pradesh (Agarkar and Agarkar, 1977); Tamil Nadu (Perumal and Anand, 2008); Uttar Pradesh (Chaturvedi et al., 1987).

22. Cosmarium granatum Breb. var. nordstedtii Hansg. (pl. 1, fig. 12)

Suxena et al., 1973. p. 326, figs. 45a, b.

L: 31-39 $\mu \mathrm{m}, \mathrm{B}: 23.3-27.2 \mu \mathrm{m}$. Distribution: Kerala

(Suxena et al., 1973).

23. Cosmarium impressulum Elfv. (pl. 1, fig. 9)

West and West, 1908. vol. 3, p. 86, pl. 72, figs. 14-18.
L: 23.3-27.2 $\mu \mathrm{m}, \mathrm{B}: 17.5-19.5 \mu \mathrm{m}$. Distribution: Kerala (Sindhu and Panikkar, 1995); Andhra Pradesh (Suxena and Venkareswarlu, 1966); East India (Turner, 1892); Gujarat (Asokakumar and Patel, 1988); Maharashtra (Dhande and Jawale, 2009; Kamat, 1975a); Uttar Pradesh (Chaturvedi et al., 1987).

24. Cosmarium indentatum Gronbl. var. ellipticum Scott and Gronbl. (pl. 1, fig. 20)

Scott and Prescott, 1961. p. 59, pl. 27, fig. 7. L: 29.2-31 $\mu \mathrm{m}, \mathrm{B}: 19.5-23.3 \mu \mathrm{m}$.

25. Cosmarium javanicum Nordst. (pl. 1, fig. 28)

Suxena and Venkateswarlu, 1966. p. 57, pl. 2, fig. 34. L: 105-124.5 $\mu \mathrm{m}, \mathrm{B}: 60.3-67 \mu \mathrm{m}$. Distribution: Kerala (Shaji et al., 1988; Suxena et al., 1973); Andhra Pradesh (Suxena and Venkareswarlu, 1966); Madhya Pradesh (Agarkar and Agarkar, 1977); Uttar Pradesh (Chaturvedi et al., 1987).

26. Cosmarium lundellii Delp. (pl. 1, fig. 23)

Scott and Prescott, 1961. p. 60, pl. 25, fig. 6.

L: 54.5-77.8 $\mu \mathrm{m}, \mathrm{B}:$ 58.4-68 $\mu \mathrm{m}$. Distribution: Kerala (Shaji et al., 1988); Andhra Pradesh (Suxena and Venkareswarlu, 1966); Gujarat (Asokakumar and Patel, 1988); Karnataka (Isaacs and Hegde, 1986); Tamil Nadu (Perumal and Anand, 2008); Uttar Pradesh (Chaturvedi et al., 1987).

27. Cosmarium lundellii Delp. var. corruptum (Turn.) West and West (pl. 1, fig. 26)

Scott and Prescott, 1961.p. 61, pl. 25, fig. 9.

L: 42.8-66 $\mu \mathrm{m}, \mathrm{B}: 39-58.4 \mu \mathrm{m}$. Distribution: Kerala (Shaji and Patel, 1990); Gujarat (Asokakumar and Patel, 1988); Maharashtra (Kamat, 1975a); Madhya Pradesh (Agarkar and Agarkar, 1977); Uttar Pradesh (Tiwari and Chauhan, 2007).

28. Cosmarium lundellii Delp. var. ellipticum West (pl. 1, fig. 27)

Scott and Prescott, 1961. p. 61, pl. 25, fig. 8.

L: 59.3-68 $\mu \mathrm{m}, \mathrm{B}: 47.7-53.5 \mu \mathrm{m}$. Distribution: Andhra Pradesh (Suxena and Venkareswarlu, 1966); Gujarat (Asokakumar and Patel, 1988); Maharashtra (Kamat, 1975a); Uttar Pradesh (Chaturvedi et al., 1987).

29. Cosmarium maculatiforme Schmidle (pl. 1, fig. 33)

Shaji and Patel, 1990. fig. 2/1.

L: 109-120.6 $\mu \mathrm{m}, \mathrm{B}: 62.2-66 \mu \mathrm{m}$. Distribution: Kerala (Shaji and Patel, 1990); Gujarat (Asokakumar and Patel, 1990).

30. Cosmarium maculatum Turn. (pl. 1, fig. 35)

Scott and Prescott, 1961. p. 61, pl. 24, fig. 2.

L: 171-190.6 $\mu \mathrm{m}, \mathrm{B}: 74-81.7 \mu \mathrm{m}$. Distribution: East India (Turner, 1892); Maharashtra (Kamat, 1975a); Tamil Nadu (Perumal and Anand, 2008).

31. Cosmarium manipurence Bruhl et Biswas (pl. 1, fig. 25) Sindhu and Panikkar, 1995. fig. 4/4. 
L: 39-50.6 $\mu \mathrm{m}$, B: 27.2-35 $\mu \mathrm{m}$. Distribution: Kerala (Sindhu and Panikkar, 1995); Uttar Pradesh (Tiwari and Chauhan, 2007).

32. Cosmarium margaritatum (Lund.) Roy and Biss. fa. minor (Boldt) W. and G.S. West (pl. 1, fig. 24)

West and West, 1912. vol. 4, p. 18, pl. 99, fig. 9.

L: 46.7-54.5 $\mu \mathrm{m}, \mathrm{B}: 34-41 \mu \mathrm{m}$. Distribution: Maharashtra (Kamat, 1975a).

33. Cosmarium margaritatum (Lund.) Roy and Biss. var. sublatum (Nordst.) Krieg. (pl. 1, fig. 36)

Scott and Prescott, 1961. p. 63, pl. 29, fig. 4.

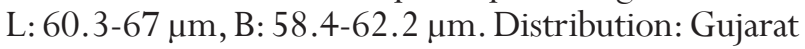
(Asokakumar and Patel, 1988); Karnataka (Isaacs and Hegde, 1986).

34. Cosmarium meneghinii Breb. (pl. 1, fig. 15)

West andWest, 1908. vol. 3, p. 90, pl. 72, figs. 29-32. L: 16.5-18.5 $\mu \mathrm{m}, \mathrm{B}: 9.7-12.6 \mu \mathrm{m}$. Distribution: Kerala (Sindhu and Panikar, 1994; Suxena et al., 1973); Andhra Pradesh (Suxena andVenkareswarlu, 1966); Bihar (Saha and Choudhary, 1984); Gujarat (Asokakumar and Patel, 1990); Maharashtra (Freitas and Kamat, 1979; Kamat, 1975b); Uttar Pradesh (Chaturvedi et al., 1987).

35. Cosmarium nitidulum De Not (pl. 1, fig. 29)

West and West, 1905. vol. 2, p. 197, pl. 64, figs. 1-3. L: 35-42.8 $\mu \mathrm{m}, \mathrm{B}: 25.3-39 \mu \mathrm{m}$. Distribution: Kerala (Suxena et al., 1973); Gujarat (Asokakumar and Patel, 1988); Maharashtra (Kamat, 1975b); Tamil Nadu (Perumal and Anand, 2008).

36. Cosmarium notabile Breb. (pl. 1, fig. 31)

West andWest, 1908. vol. 3, p. 15, pl. 66, figs. 15-16. L: 23.3-31 $\mu \mathrm{m}, \mathrm{B}: 19.5-24.3 \mu \mathrm{m}$. Distribution: Kerala (Sindhu and Panikkar, 1994).

37. Cosmarium nudum (Turn.) Gutw. (pl. 1, fig. 38) Scott and Prescott, 1961. p. 63, pl. 30, figs. 1, 2. L: 46.7-48.6 $\mu \mathrm{m}, \mathrm{B}: 43.8-45.7 \mu \mathrm{m}$. Distribution: Kerala (Shaji and Patel, 1990); Uttar Pradesh (Chaturvedi et al., 1987).

38. Cosmarium obsoletum (Hantzsch) Reinsch (pl. 1, fig. 37) Scott and Prescott, 1961. p. 63, pl. 26, fig. 1.

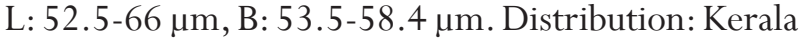
(Jose and Francis, 2007); East India (Turner, 1892); Karnataka (Isaacs and Hegde, 1986); M.P. (Agarkar et al., 1983); Tamil Nadu (Perumal and Anand, 2008); Uttar Pradesh (Tiwari and Chauhan, 2007).

39. Cosmarium obsoletum (Hantzsch) Reinsch var. sitvense Gutw. (pl. 1, fig. 39)

Scott and Prescott, 1961. p. 63, pl. 25, fig. 11 and pl. 26, fig. 2.

L: 62.2-66 $\mu \mathrm{m}, \mathrm{B}: 64.2-77.8 \mu \mathrm{m}$. Distribution: Kerala (Sindhu and Panikkar, 1995; Suxena et al., 1973); Bihar (Das et al., 1990; Saha and Choudhary, 1984);
Chhattisgarh (Toppo and Suseela, 2009); Maharashtra (Kamat, 1975a); Karnataka (Hegde and Isaacs, 1988); Madhya Pradesh (Agarkar et al., 1979).

40.Cosmarium pachydermum Lund. (pl. 1, fig. 41)

Scott and Prescott, 1961.p. 64, pl. 25, fig. 5.

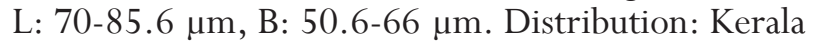
(Sindhu and Panikkar, 1994).

41. Cosmarium portianum Arch. (pl. 1, fig. 30)

Scott and Prescott, 1961. p. 65, pl. 28, fig. 8.

L: 23.3-27.2 $\mu \mathrm{m}, \mathrm{B}: 17.5-19.5 \mu \mathrm{m}$. Distribution:

Kerala (Jose and Francis, 2007, Shaji and Patel,1991); East India (Turner, 1892); Bihar (Das et al., 1990); Gujarat (Asokakumar and Patel, 1988); Maharashtra (Freitas and Kamat, 1979; Kamat, 1975a; Kamat, 1975b); Madhya Pradesh (Agarkar et al., 1983); Tamil nadu (Perumal and Anand, 2008).

42. Cosmarium pseudobroomei Wolle (pl. 1, fig. 32)

West and West, 1912. vol. 4, p. 22, pl. 100, figs. 7, 8 . L: 31-35 $\mu \mathrm{m}, \mathrm{B}: 30-32 \mu \mathrm{m}$. Distribution: Kerala (Shaji and Patel, 1990); Andhra Pradesh (Suxena and Venkareswarlu, 1966); East India (Turner, 1892); Maharashtra (Kamat, 1975b); Tamil Nadu (Perumal and Anand, 2008).

43. Cosmarium pseudoconnatum Nordst. var. constrictum West (pl. 1, fig. 54)

West and West, 1908. vol. 3, p. 29, pl. 67, figs. 23. L: 60.3-72 $\mu \mathrm{m}, \mathrm{B}: 40-48.6 \mu \mathrm{m}$.

44. Cosmarium pseudopyramidatum Lund. var. oculatum Krieg. (pl. 1, fig. 40)

Scott and Prescott, 1961. p. 67, pl. 27, figs. 2, 3.

L: 54.5-61.2 $\mu \mathrm{m}, \mathrm{B}:$ 34-37 $\mu \mathrm{m}$. Distribution: Kerala (Sindhu and Panikkar, 1995); Andhra Pradesh (Suxena and Venkareswarlu, 1966); Madhya Pradesh (Agarkar et al., 1983).

45. Cosmarium pseudotoxicandrum Nordst. (pl. 1, fig. 34) Croasdale and Gronblad, 1964. p. 184, pl. 13, fig. 20. L: 33-39 $\mu \mathrm{m}, \mathrm{B}$ : 36-39 $\mu \mathrm{m}$. Distribution: Tamil Nadu (Perumal and Anand 2008).

46. Cosmarium punctulatum Breb. var. subpunctulatum (Nordst.) Borg. (pl. 1, fig. 42)

West and West, 1908. vol. 3, p. 209, pl. 84, figs. 15-20 and pl. 85, figs. 1-3.

L: 31-39 $\mu \mathrm{m}, \mathrm{B}: 27.2-33 \mu \mathrm{m}$. Distribution: Kerala (Shaji et al., 1988); Gujarat (Asokakumar and Patel, 1988); Madhya Pradesh (Das et al., 1990; Saha and Choudhary, 1984).

47. Cosmarium pygmaeum Arch. (pl. 1, fig. 46)

West and West, 1908. vol. 3, p. 73, pl. 71, figs. 22-31. L: 7.8-11.7 $\mu \mathrm{m}, \mathrm{B}: 9.7-13.6 \mu \mathrm{m}$. Distribution: Kerala (Sindhu and Panikkar, 1994); Gujarat (Asokakumar and Patel, 1988).

48. Cosmarium pyramidatum Breb. (pl. 1, fig. 47) 
West and West, 1905. vol. 2, p. 199, pl. 64, figs. 5-7. L: 97.3-116.7 $\mu \mathrm{m}, \mathrm{B}:$ 52.5-58.4 $\mu \mathrm{m}$. Distribution: Bihar (Das et al., 1990); Maharashtra (Kamat, 1975a); Madhya Pradesh (Agarkar et al., 1979; Agarkar and Agarkar, 1977).

49. Cosmarium pyramidatum Breb. var. stephanii (IreneeMarie) Krieg. et Gerloff (pl. 1, fig. 48)

Shaji et al., 1989. pl. 2, fig. 7.

L: 60.3-66 $\mu \mathrm{m}$, B: 36-40.8 $\mu \mathrm{m}$. Distribution: Kerala (Shaji et al., 1989); Karnataka (Gurudeva et al., 1983).

50. Cosmarium quadrifarium Lund. (pl. 1, fig. 43)

West and West, 1908. vol. 3, p. 141, pl. 76, figs. 15-17 and $\mathrm{pl}$. 77, figs. 1-3.

L: $40-42.8 \mu \mathrm{m}, \mathrm{B}: 30-33 \mu \mathrm{m}$.

51. Cosmarium quadri verrucosum West and West var. undulatum Scott and Prescott (pl. 1, fig. 45)

Scott and Prescott, 1961. p. 67, pl. 30, fig. 7.

L: 24.3-35 $\mu \mathrm{m}, \mathrm{B}: 19.5-24.3 \mu \mathrm{m}$.

52. Cosmarium quadrum Lund. (pl. 1, fig. 51)

West and West, 1912. vol. 4, p. 20, pl. 100, figs. 3-6. L: 46.7-54.5 $\mu \mathrm{m}, \mathrm{B}: 55.4-59.3 \mu \mathrm{m}$. Distribution: Kerala (Shaji et al., 1988; Sindhu and Panikar, 1994); Bihar (Das et al., 1990); Maharashtra (Kamat, 1975a); M.P. (Agarkar and Agarkar, 1977); T.N. (Perumal and Anand, 2008); U.P. (Chaturvedi et al., 1987).

53. Cosmarium reniforme (Ralfs) Arch. (pl. 1, fig. 52)

West and West, 1908. vol. 3, p. 157, pl. 79, figs. 1-2. L: 35-48.6 $\mu \mathrm{m}, \mathrm{B}: 33-44.7 \mu \mathrm{m}$. Distribution: Bihar (Das et al., 1990; Saha and Choudhary, 1984); Maharashtra (Dhande and Jawale, 2009; Kamat, 1975a); Tamil Nadu (Perumal and Anand, 2008); Uttar Pradesh (Tiwari and Chauhan, 2007).

54. Cosmarium retusiforme (Wille) Gutw. (pl. 1, fig. 60)

Scott and Prescott, 1961. p. 68, pl. 32, fig. 15.

L: 23.3-25.3 $\mu \mathrm{m}, \mathrm{B}: 19.5-23.3 \mu \mathrm{m}$. Distribution:

Kerala (Shaji and Patel, 1990); Gujarat (Asokakumar and Patel, 1988); Karnataka (Isaacs and Hegde, 1986);

Tamil Nadu (Perumal and Anand, 2008).

55.Cosmarium scabrum Turn. (pl. 1, fig. 55)

Scott and Prescott, 1961. p. 68, pl. 29, fig. 3.

L: 46.7-51.5 $\mu \mathrm{m}, \mathrm{B}: 47.7-53.5 \mu \mathrm{m}$. Distribution: East India (Turner, 1892); Karnataka (Gurudeva et al., 1983); Uttar Pradesh (Chaturvedi et al., 1987).

56. Cosmarium speciosum Lund. (pl. 1, fig. 50)

West and West, 1908. vol. 3, p. 247, pl. 89, figs. 1-3. L: 48.6-62.2 $\mu \mathrm{m}, \mathrm{B}: 31-40 \mu \mathrm{m}$. Distribution: Tamil Nadu (Perumal and Anand, 2008); Uttar Pradesh (Tiwari and Chauhan, 2007).

57. Cosmarium spinuliferum West and West (pl. 1, fig. 63)

Scott and Prescott, 1961. p. 69, pl. 29, figs. 6, 7.

L: 31-34 $\mu \mathrm{m}, \mathrm{B}: 32-35 \mu \mathrm{m}$. Distribution: Maharashtra (Kamat, 1975b).
58. Cosmarium striolatum Nag. (pl. 1, fig. 56)

Scott and Prescott, 1961. p. 70, pl. 25, figs. $2,3$.

L: 93.4-112.8 $\mu \mathrm{m}, \mathrm{B}:$ 54.5-62.2 $\mu \mathrm{m}$. Distribution:

Maharashtra (Kamat, 1975a); Madhya Pradesh (Agarkar et al., 1983).

59. Cosmarium subcostatum Nordst. (pl. 1, fig. 65)

West and West, 1908. vol. 3, p. 236, pl. 87, figs. 3-5. L: 27.2-35 $\mu \mathrm{m}, \mathrm{B}: 24.3-27.2 \mu \mathrm{m}$. Distribution: Kerala (Shaji et al., 1989); Uttar Pradesh (Chaturvedi et al., 1987).

60. Cosmarium subcrenatum Hantzsch (pl. 1, fig. 64)

West and West, 1908. vol. 3, p. 228, pl. 86, figs. 10-14. L: 26.3-39 $\mu \mathrm{m}, \mathrm{B}: 22.4-31 \mu \mathrm{m}$. Distribution: Chhattisgarh (Toppo and Suseela, 2009); Maharashtra (Freitas and Kamat, 1979); Tamil Nadu (Perumal and Anand, 2008); Uttar Pardesh (Chaturvedi et al., 1987).

61. Cosmarium subspeciosum Nordst. var. validius Nordst. (pl. 1, fig. 57)

West and West, 1908. vol. 3, p. 253, pl. 89, figs. 12, 13.

L: 81.7-93.4 $\mu \mathrm{m}, \mathrm{B}:$ 60.3-66 $\mu \mathrm{m}$. Distribution: Kerala: (Sindhu and Panikkar, 1995); Bihar (Saha and Choudhary, 1984); Chhattisgarh (Toppo and Suseela, 2009); Karnataka (Isaacs and Hegde, 1986); Maharashtra (Kamat, 1975b); U.P. (Chaturvedi et al., 1987).

62. Cosmarium subturgidum (Turn.) Schmidle (pl. 1, fig. 61) West and West, 1908. vol. 3, p. 116, pl. 75, fig. 5.

L: $175-182.8 \mu \mathrm{m}, \mathrm{B}: 85.6-97.3 \mu \mathrm{m}$.

63. Cosmarium taxichondrum Lund. var. nudum Turn. (pl. 1, fig. 67)

Turner, 1892. p. 60, pl. 8, fig. 64 .

L: 39-41.8 $\mu \mathrm{m}, \mathrm{B}: 42.8-44.7 \mu \mathrm{m}$. Distribution: East India (Turner 1892).

64. Cosmarium tjibenongense Gutw. fa. minus G.S. West (pl. 1, fig. 62)

Scott and Prescott, 1961.p. 72, pl. 27, fig. 8.

L: 31-34 $\mu \mathrm{m}, \mathrm{B}: 20.4-22.4 \mu \mathrm{m}$. Distribution:

Karnataka (Hegde, 1986; Hegde and Isaacs, 1988).

65. Cosmarium trachypleurum Lund. var. nordstedtii Gutw. (pl. 1, fig. 59)

Scott and Prescott, 1961.p. 72, pl. 31, fig. 6.

L: 31-36 $\mu \mathrm{m}, \mathrm{B}: 27.2-31 \mu \mathrm{m}$. Distribution: Kerala (Shaji et al., 1989); Karnataka (20); Maharashtra (Kamat, 1975a).

66. Cosmarium tumidum Lund. (pl. 1, fig. 58)

Scott and Prescott, 1961. p. 72, pl. 27, fig. 16.

L: 31-34 $\mu \mathrm{m}, \mathrm{B}: 24.3-27.2 \mu \mathrm{m}$. Distribution:

Maharashtra (Kamat, 1975a).

67. Cosmarium turgidum Breb. (pl. 1, fig. 66)

West and West, 1908. vol. 3, p. 115, pl. 75, figs. 1-3. L: 171-210 $\mu \mathrm{m}, \mathrm{B}: 77.8-81.7 \mu \mathrm{m}$. Distribution: 
Table 1: Seasonal and spatial variation of genus Cosmarium Corda in the Kole lands of Thrissur, Kerala

\begin{tabular}{|c|c|c|c|c|c|c|c|c|c|c|c|c|c|c|}
\hline \multirow{2}{*}{$\begin{array}{l}\text { Serial } \\
\text { number }\end{array}$} & \multirow[t]{2}{*}{ Name of taxa } & \multicolumn{3}{|c|}{ Seasons } & \multicolumn{10}{|c|}{ Stations } \\
\hline & & $\mathrm{Si}$ & S ii & S iii & 1 & 2 & 3 & 4 & 5 & 6 & 7 & 8 & 9 & 10 \\
\hline 1 & C. apertum & - & - & + & + & - & - & - & - & - & - & - & - & $\overline{-}$ \\
\hline 2 & C. arnellii & + & + & - & - & + & - & - & + & - & - & + & - & - \\
\hline 3 & C. auriculatum & + & + & + & - & + & + & + & + & + & + & + & + & + \\
\hline 4 & C. binum & + & - & + & - & - & - & - & - & + & - & - & + & + \\
\hline 5 & C. blyttii & + & + & + & - & + & - & - & + & - & + & - & - & + \\
\hline 6 & C. brebissonii & + & - & - & + & - & - & - & - & - & - & - & - & + \\
\hline 7 & C. circulare & + & + & + & + & + & + & + & - & - & - & + & - & - \\
\hline 8 & C. clepsydra var. duplex & + & - & + & + & - & + & - & - & - & - & - & - & - \\
\hline 9 & C. commissurale & + & - & - & - & - & - & + & - & - & - & - & - & - \\
\hline 10 & C. connatum & + & + & + & + & + & + & + & + & - & - & + & + & - \\
\hline 11 & C. contractum & + & + & + & + & + & - & - & - & + & + & - & - & - \\
\hline 12 & C. contractum var. ellipsoideum & + & + & + & + & + & + & - & + & - & - & - & - & - \\
\hline 13 & C. contractum var. incrassatum & - & + & + & - & - & - & + & - & - & - & + & + & - \\
\hline 14 & C. contractum var. minutum & + & + & + & + & + & + & + & + & - & - & - & - & + \\
\hline 15 & C. cuneatum & + & + & + & + & + & + & + & + & + & + & + & - & + \\
\hline 16 & C. cyclicum & + & + & - & + & - & - & + & - & - & + & - & - & - \\
\hline 17 & C. decoratum var. dentiferum & + & + & + & - & + & - & - & + & - & - & - & + & - \\
\hline 18 & C. depressum & - & + & - & - & - & - & + & + & - & + & - & - & - \\
\hline 19 & C. exiguum & + & + & + & + & + & _- & - & - & - & - & - & - & - \\
\hline 20 & C. geminatum var. ornatum & - & + & + & + & + & - & + & - & - & + & - & - & - \\
\hline 21 & C. granatum & + & + & + & + & + & _- & + & + & + & - & + & + & + \\
\hline 22 & C. granatum var. nordstedtii & + & + & + & + & - & + & - & + & - & + & + & - & - \\
\hline 23 & C. impressulum & + & + & + & + & + & - & + & + & - & - & - & - & - \\
\hline 24 & C. indentatum var. ellipticum & + & + & + & + & - & - & + & - & + & - & + & - & - \\
\hline 25 & C. javanicum & - & - & + & - & + & - & - & - & _- & - & - & - & - \\
\hline 26 & C. Iundellii & + & + & + & + & + & + & + & + & + & + & + & - & + \\
\hline 27 & C. Iundellii var. corruptum & + & + & + & + & + & + & + & - & - & - & - & + & - \\
\hline 28 & C. Iundellii var. ellipticum & - & + & + & + & + & - & - & + & - & + & - & + & + \\
\hline 29 & C. maculatiforme & + & + & + & - & - & + & - & + & - & - & - & - & - \\
\hline 30 & C. maculatum & + & + & + & + & - & + & - & + & - & + & - & - & - \\
\hline 31 & C. manipurence & + & + & + & - & + & - & - & - & - & + & - & - & + \\
\hline 32 & C. margaritatum fa. minor & + & + & + & - & - & - & + & + & - & - & + & - & + \\
\hline 33 & C. margaritatum var. sublatum & + & + & + & + & - & - & - & + & - & - & - & - & + \\
\hline 34 & C. meneghinii & - & + & - & - & - & - & + & - & - & - & - & - & - \\
\hline 35 & C. nitidulum & - & + & - & - & - & - & - & + & - & - & - & - & - \\
\hline 36 & C. notabile & + & - & - & - & - & + & - & - & - & - & - & - & - \\
\hline 37 & C. nudum & - & + & + & - & - & - & - & + & - & + & - & - & - \\
\hline 38 & C. obsoletum & - & + & - & - & - & - & - & + & - & - & - & - & - \\
\hline 39 & C. obs oletum var. sitvense & - & - & + & - & - & - & - & - & - & - & + & - & - \\
\hline 40 & C. pachydermum & + & - & - & - & - & - & + & - & - & - & - & - & - \\
\hline 41 & C. portianum & + & + & - & + & - & - & - & - & - & + & - & + & - \\
\hline 42 & C. pseudobroomei & - & + & + & + & - & _- & - & - & + & - & + & + & + \\
\hline 43 & C. pseudoconnatum var. constrictum & - & - & + & - & - & - & - & - & - & - & - & - & + \\
\hline 44 & C. pseudopyramidatum var. oculatum & + & - & - & - & - & - & - & + & - & - & - & - & + \\
\hline 45 & C. pseudotoxicandrum & - & - & + & - & - & - & + & - & - & - & - & - & - \\
\hline 46 & C. punctulatum var. subpunctulatum & + & + & + & + & + & - & + & + & + & - & - & + & + \\
\hline 47 & C. pygmaeum & + & + & + & + & + & + & - & - & - & - & - & - & - \\
\hline 48 & C. pyramidatum & - & - & + & + & - & - & - & - & - & - & - & - & - \\
\hline 49 & C. pyramidatum var. stephanii & - & + & - & + & - & - & - & - & - & - & - & - & - \\
\hline 50 & C. quadrifarium & + & + & + & + & - & + & + & - & - & + & + & - & - \\
\hline 51 & C. quadriverrucosum var. undulatum & - & - & + & - & - & + & - & - & - & - & - & - & - \\
\hline 52 & C. quadrum & + & + & + & + & + & + & + & + & - & + & + & + & - \\
\hline 53 & C. reniforme & + & + & - & - & - & - & - & - & + & - & - & - & + \\
\hline 54 & C. retusiforme & - & + & + & + & + & + & + & + & - & + & - & - & - \\
\hline 55 & C. scabrum & - & + & + & - & + & - & + & + & - & - & - & - & - \\
\hline 56 & C. speciosum & + & - & - & - & - & - & - & - & - & - & + & - & - \\
\hline 57 & C. spinuliferum & - & + & + & - & - & + & - & - & - & - & - & - & - \\
\hline 58 & C. striolatum & - & + & + & + & + & - & - & - & - & - & - & - & - \\
\hline 59 & C. subcostatum & - & + & - & - & - & - & - & - & - & + & - & + & - \\
\hline 60 & C. subcrenatum & + & + & + & + & - & + & + & + & - & + & - & + & - \\
\hline 61 & C. subspeciosum var. validius & - & + & - & - & - & - & + & - & - & - & - & - & - \\
\hline 62 & C. subturgidum & - & + & + & + & - & - & - & + & - & - & - & - & + \\
\hline 63 & C. taxichondrum var. nudum & + & - & + & - & - & - & - & + & - & - & - & - & + \\
\hline 64 & C. tjibenongense fa. minus & + & - & + & + & + & - & - & - & - & - & + & - & - \\
\hline 65 & C. trachypleurum var. nordstedtii & + & + & + & + & + & + & + & + & - & + & + & - & - \\
\hline 66 & C. tumidum & - & + & + & + & - & - & - & - & - & + & - & - & - \\
\hline 67 & C. turgidum & + & + & + & - & - & + & + & - & - & - & - & - & - \\
\hline 68 & C. vexatum & + & + & + & + & - & - & - & - & - & - & - & - & - \\
\hline
\end{tabular}

+: Present, -: Absent, S i: Pre-monsoon season (Feb-May), S ii: Monsoon season (Jun-Sep), S iii: Post-monsoon season (Oct-Jan). Stations: (1) Pullur, (2) Muriyad, (3) Nambiankavu, (4) Mapranam, (5) Chemmanda, (6) Cherpu, (7) Palakkal, (8) Chettupuzha, (9) Anthikkad, (10) Enamavu 
Maharashtra (Freitas and Kamat, 1979);T.N. (Perumal and Anand, 2008).

68. Cosmarium vexatum West (pl. 1, fig. 68)

West and West, 1908. vol. 3, p. 187, pl. 92, fig. 4.

L: 40-44.7 $\mu \mathrm{m}, \mathrm{B}: 35-39 \mu \mathrm{m}$. Distribution: Maharashtra

(Dhande and Jawale, 2009; Freitas and Kamat, 1979).

Seasonal and spatial variation of genus Cosmarium Corda in Thrissur Kole lands, Kerala were given in Table 1. The seasonal analysis of Cosmarium at different stations revealed that 29 taxa were observed throughout the year irrespective of the seasonal changes, 43 taxa were found during pre-monsoon (Feb-May), 51 taxa during monsoon (Jun-Sep) and 51 taxa during post-monsoon (Oct-Jan) seasons in the study area (Table 1). More diversity of Cosmarium was observed in station 1 (Pullur) with 37 taxa followed by station 5 (Chemmanda) with 30 taxa.

In Thrissur Kole ecosystem, the periods of cultivation are well-defined, the soils are comparatively fertile and productive, and the microclimate is favorable. The variations in rainfall and the physicochemical parameters are responsible for the fluctuations in the quality and quantity of algae in water bodies.

C. geminatum Lund. var. ornatum Behre, C. indentatum Gronbl. var. ellipticum Scott and Gronbl., C. pseudoconnatum Nordst. var. constrictum West, C. quadrifarium Lund., C. quadriverrucosum West and West var. undulatum Scott and Prescott and C. subturgidum (Turn.) Schmidle are new additions to the desmid flora of India.

\section{ACKNOWLEDGEMENT}

University Grants Commission (UGC), New Delhi is gratefully acknowledged for the FIP fellowship awarded to the first author.

\section{REFERENCES}

Agarkar DS, Agarker MS, Banerjee S. Desmids of Jabalpur, Madhya Pradesh, India. In: Cramer: Bibliotheca Phycologia. Vol. 66. Schweizerbart science publishers; 1983. p. 333-70.

Agarkar DS, Agarker MS, Dikshit R. Desmids from Bandhavgarh, Madhya Pradesh, India. Hydrobiologia 1979;65:213-23.

Agarker MS, Agarkar DS. Desmids from Pachmarhi, Madhya Pradesh, India. Hydrobiologia 1977;54:23-32.

Asokakumar CK, Patel RJ. Desmids of Gujarat - Genus Cosmarium Corda. Phykos 1988;27:117-28.

Asokakumar CK, Patel RJ. Desmids of Gujarat - I. Genus Cosmarium Corda. Phykos 1990;29:95-101.

Chaturvedi UK, Pandey UC, Habib I, Shukla HM. Desmids of bareilly - II. Phykos 1987;26:95-102.
Croasdale H, Gronblad R. Desmids of labrador 1. Desmids of the southeastern coastal area. Trans Am Microsc Soc 1964;83:142-212.

Das RN, Mahato AK, Mahato P. Desmids of Singbhum district I. Genus Cosmarium Corda. Phykos 1990;29:115-20.

Dhande JS, Jawale AK. Genus Cosmarium Corda from Hartala Lake district Jalgaon, Maharashtra. Shodh, Samiksha Aur Mulyankan 2009;2:196-8.

Freitas JF, Kamat ND. Desmidiaceae of Nagpur. Phykos 1979;18:97-103.

Gurudeva MR, Arekal GD, Somashekar RK. Desmid flora of Savandurga, Karnataka. Phykos 1983;22:48-56.

Hegde GR, Isaacs SW. Certain interesting desmid taxa from Uttara Kannada district of Karnataka State. Phykos 1988;27:8-12.

Hegde GR. Records of desmids new to Karnataka state - II Genus Cosmarium Corda. Phykos 1986;25:123-8.

Isaacs SW, Hegde GR. Freshwater algae of Uttara Kannada district, Karnataka State. Phykos 1986;25:102-7.

Johnkutty I, Venugopal VK. Kole Lands of Kerala. Thrissur: Kerala Agricultural University; 1993. p. 68.

Jose J, Francis MS. Investigation in the algal flora of Thodupuzha Thaluk, Kerala. Indian Hydrobiol 2007;10:79-86.

Kamat ND. Algae of Vidarbh, Maharashtra. J Bombay Nat Hist Soc 1975a;72:450-76.

Kamat ND. Desmids of Marathwada, Maharashtra. J Bombay Nat Hist Soc 1975b;72:616-8.

Maya S, Prameela SK, Sarojini MV. A preliminary study on the algal flora of temple tanks of southern Kerala. Phykos 2000;39:77-83.

Nurul IA, Irfanullah HM. Hydrobiological studies within the tea gardens at Srimangal, Bangladesh. V. desmids (Euastrum, Micrasterias, Actinotaenium and Cosmarium). Bangladesh J Plant Taxonomy 2006;13:1-20.

Perumal GM, Anand N. Manuel of Freshwater Algae of Tamil Nadu. Dehradun: Bishen Singh Mahendrapal Singh Publishers; 2008. p. 133.

Saha LC, Choudhary SK. Desmids of Bhagalpur, Bihar. J Econ Tax Bot 1984;5:987-8.

Scott AM, Prescott GW. Indonesian desmids. Hydrobiologia 1961;17:1-132.

Shaji C, Jose L, Patel RJ. Additions to desmid flora of Kerala. Phykos 1988;27:32-7.

Shaji C, Jose L, Patel RJ. Contribution to desmid flora of Kerala, India. Nova Hedwigia 1989;49:169-82.

Shaji C, Patel RJ. Contribution to desmid flora of Kerala, India - II. Nova Hedwigia 1991;52:197-225.

Shaji C, Patel RJ. Desmids new to Kerala. Feddes Repertorium 1990;101:277-84.

Sheeba S, Ramanujan N. Phytoplankton composition and distribution in Ithikkara River, Kerala. Indian Hydrobiol 2005;8:11-7. 
Sindhu P, Panikkar MV. Desmid flora of Quilon, Kerala. Cosmarium Corda. J Econ Tax Bot 1994;18:711-4.

Sindhu P, Panikkar MV. Desmids new to Kerala, India - 1. Feddes Repertorium 1995;106:317-23.

Suxena MR, Venkateswarlu V, Raju NS, Rao VS. The algae and testacea of Cranganore, Kerala State, India. J Indian Bot Soc 1973;52:316-41.

Suxena MR, Venkateswarlu V. Desmids of Andhra Pradesh, I. from Pakhal Lake, Warangal. Hydrobiologia 1966;28:49-65.

Tiwari A, Chauhan SV. Species diversity and seasonal variation of desmids in Agra city. Indian Hydrobiol 2007;10:307-17.

Toppo K, Suseela MR. Cosmarium diversity of Mani Pokhar Pond of Jashpur District in Chhattisgarh State, India. Ann
Forestry 2009;17:117-24.

Turner WB. The Fresh-Water Algae (Principally Desmidieae) of East India. Kongl. Svenska Vetenskaps-Akademiens Handlingar. Vol. 25. No. 5. Stockholm: Norstedt: Norstedt; 1892. p. 1-187, Plates I-XXIII.

West W, West GS. A Monograph of the British Desmidiaceae. Vol. 2. London:The Ray Society; 1905. p. 125-206, Plates 56-64.

West W, West GS. A Monograph of the British Desmidiaceae. Vol. 3. London:The Ray Society; 1908. p. 274, Plates 65-95.

West W, West GS. A Monograph of the British Desmidiaceae. Vol. 4. London: The Ray Society; 1912. p. 1-48, Plates 96-103. 